\title{
A VARIATIONAL PRINCIPLE FOR TWO-FLUID MODELS
}

\author{
Sergey Gavrilyuk, Henri Gouin and Yurii Perepechko
}

S.G. and H.G.: L. M. M. T. Box 322, University of Aix-Marseille

Avenue Escadrille Normandie-Niemen, 13397 Marseille Cedex 20 France

Y.P.: United Institute of Geology, Geophysics and Mineralogy, Siberian Branch of the Russian Academy of Sciences, Novisibirsk 630090, Russia

E-mail: henri.gouin@univ-cezanne.fr

\begin{abstract}
A variational principle for two-fluid mixtures is proposed. The Lagrangian is constructed as the difference between the kinetic energy of the mixture and a thermodynamic potential conjugated to the internal energy with respect to the relative velocity of phases. The equations of motion and a set of RankineHugoniot conditions are obtained. It is proved also that the convexity of the internal energy guarantees the hyperbolicity of the one-dimensional equations of motion linearized at rest.
\end{abstract}

1 - Variational approach to the description of homogenous two-velocity media

The variational approach to the construction of two-fluid models was used by many authors (A.Bedford \& D.S. Drumheller (1978), V.L.Berdichevsky (1983), J.A. Geurst $(1985,1986)$, H. Gouin (1990)). Here we give its generalisation for the case of homogeneous two-fluid mixtures. A physical example of such flow is a motion of a mixture of two gases with quite different molecular weights. The Hamilton's principle is applied for perfect fluid motions. In order to obtain the equations of motion of the mixture from this variational principle, we neglect the dissipative effects. We will then consider only mechanical processes by suppressing thermal evolution. We suppose that the homogeneous 
mixture motion is well represented by the velocities of its components $\vec{u}_{1}, \vec{u}_{2}$, the average densites $\rho_{1}, \rho_{2}$ and the total internal energy $U$. The total energy of a two-velocity medium is written in the form:

$$
E=\frac{\rho_{1}\left|\vec{u}_{1}\right|^{2}}{2}+\frac{\rho_{2}\left|\vec{u}_{2}\right|^{2}}{2}+U
$$

In order to define the internal energy of one-velocity media, a moving coordinate system, where the elementary volume of the continuum is at rest, is considered. The total energy of the continuum with respect to this system is called the internal energy of the motion. For a two-velocity medium, there is no coordinate system, within the framework of which any motion could be disregarded. This is the reason why the standard definition of internal energy leads to its dependence on the relative motion of components.

Let $\vec{w}=\vec{u}_{2}-\vec{u}_{1}, w=|\vec{w}|$. We propose the following extended form of Hamilton's principle of least action for the two-velocity systems:

$$
\delta I=0, I=\int_{t_{1}}^{t_{2}} \int_{\mathcal{D}}\left(\rho_{1} \frac{\left|\vec{u}_{1}\right|^{2}}{2}+\rho_{2} \frac{\left|\vec{u}_{2}\right|^{2}}{2}-W\left(\rho_{1}, \rho_{2}, w\right)\right) d \vec{x} d t
$$

with the kinematic contraints

$$
\frac{\partial \rho_{i}}{\partial t}+\operatorname{div}\left(\rho_{i} \vec{u}_{i}\right)=0
$$

Here $\left[t_{1}, t_{2}\right]$ is a time interval, $\mathcal{D}$ is a domain in the physical space, the potential $W\left(\rho_{1}, \rho_{2}, w\right)$ is connected with the internal energy $U$ by the partial Legendre transformation with respect to the variable $w$ :

$$
U\left(\rho_{1}, \rho_{2}, i\right)=W\left(\rho_{1}, \rho_{2}, w\right)-w \frac{\partial W}{\partial w}=W+i w, \quad \text { with } \quad i=-\frac{\partial W}{\partial w} .
$$

The internal energy of the medium $U\left(\rho_{1}, \rho_{2}, i\right)$ is generally a convex function of its variables. This assumption corresponds to the condition of thermodynamic stability. As a consequence, $W\left(\rho_{1}, \rho_{2}, w\right)$ is a convex function with respect to variables $\rho_{1}, \rho_{2}$ and a concave function with respect to variable $w$. A simple case is associated with

$$
W\left(\rho_{1}, \rho_{2}, w\right)=\varepsilon\left(\rho_{1}, \rho_{2}\right)-\frac{a\left(\rho_{1}, \rho_{2}\right) w^{2}}{2},
$$




$$
U\left(\rho_{1}, \rho_{2}, i\right)=\varepsilon\left(\rho_{1}, \rho_{2}\right)+\frac{i^{2}}{2 a\left(\rho_{1} \rho_{2}\right)},
$$

where $\varepsilon\left(\rho_{1}, \rho_{2}\right)$ is a convex function with respect to $\rho_{1}, \rho_{2}$ and $a\left(\rho_{1}, \rho_{2}\right)$ is a positive function. If the relative velocity $\vec{w}$ is small enough, the energy $U$ is convex. The example (1.4) is a reasonable approximation of a general case as $W$ is an analytic function of the velocities $\vec{u}_{i}$ (and hence, an analytic function of $w^{2}$ only). The term in equation (1.4) which is quadratic with respect to the relative velocity $\vec{w}$, can be considered as the energy due to an added mass effect. We note that this quadratic dependence is usually used in the theory of bubbly liquids (V.L. Berdichevsky, 1983, J.A. Geurst, 1985, 1986). To derive the governing equations and the Rankine-Hugoniot conditions, it is not necessary to focus on the particular case (1.4).

\section{2 - Governing equations}

We introduce Lagrange coordinates $\vec{X}_{i}$ for each component:

$$
\frac{d_{i} \vec{X}_{i}}{d t}=0, \quad \text { where } \quad \frac{d_{i}}{d t}=\frac{\partial}{\partial t}+\left(\vec{u}_{i} \nabla\right), \quad i=1,2 .
$$

It follows from (2.1) that

$$
\vec{u}_{i}=-\left(\frac{\partial \vec{X}_{i}}{\partial \vec{x}}\right)^{-1}<\frac{\partial \vec{X}_{i}}{\partial t}>,
$$

where the operation $A<\vec{f}>$ denotes the product of the tensor $A$ by the vector $\vec{f}$.

Introducing the Lagrange multipliers $\varphi_{1}(t, \vec{x}), \varphi_{2}(t, \vec{x})$, corresponding to the balance of masses (1.2), we consider the Lagrangian $L$ of the system

$$
L=\rho_{1}\left(\frac{1}{2}\left|\vec{u}_{1}\right|^{2}-\frac{d_{1} \varphi_{1}}{d t}\right)+\rho_{2}\left(\frac{1}{2}\left|\vec{u}_{2}\right|^{2}-\frac{d_{2} \varphi_{2}}{d t}\right)-W\left(\rho_{1}, \rho_{2}, w\right) .
$$

The formulae $(2.2),(2.3)$ give the Lagrangian $L$ as a function of variables $\frac{\partial \vec{X}_{i}}{\partial t}$, $\frac{\partial \vec{X}_{i}}{\partial \vec{x}}, \frac{\partial \varphi_{i}}{\partial t}, \frac{\partial \varphi_{i}}{\partial \vec{x}}, \rho_{1}, \rho_{2}$. Calculating the corresponding variational derivatives, we find the governing equations of motion :

$$
\frac{d_{i} \vec{K}_{i}}{d t}+\frac{\partial \vec{u}_{i}^{*}}{\partial \vec{x}}<\vec{K}_{i}-\vec{u}_{i}>+\nabla^{*}\left(\frac{\partial W}{\partial \rho_{i}}\right)=0
$$




$$
\vec{K}_{i}=\vec{u}_{i}-(-1)^{i} \frac{1}{\rho_{i}} \frac{\partial W}{\partial w} \frac{\vec{w}}{w}, i=1,2
$$

and, of course, the mass conservation laws (1.2). Here and later "*" denotes the transposition. For the case (1.4) equations (2.4) have the form :

$$
\begin{gathered}
\frac{d_{i} \vec{K}_{i}}{d t}+(-1)^{i} \frac{a\left(\rho_{1}, \rho_{2}\right)}{\rho_{i}} \frac{\partial \vec{u}_{i}{ }^{*}}{\partial \vec{x}}<\vec{w}>+\nabla^{*}\left(\frac{\partial \varepsilon}{\partial \rho_{i}}-\frac{1}{2} w^{2} \frac{\partial a}{\partial \rho_{i}}\right)=0, \\
\vec{K}_{i}=\vec{u}_{i}+(-1)^{i} \frac{a\left(\rho_{1}, \rho_{2}\right)}{\rho_{i}} \vec{w} .
\end{gathered}
$$

The system (1.2), (2.4) yields the momentum conservation law and the energy conservation law, corresponding to the homogeneity of the Lagrangian with respect to space and time variables:

$$
\begin{gathered}
\frac{\partial}{\partial t}\left(\rho_{1} \vec{u}_{1}+\rho_{2} \vec{u}_{2}\right)+\operatorname{div}\left(\rho_{1} \vec{u}_{1} \otimes \vec{u}_{1}^{*}+\rho_{2} \vec{u}_{2} \otimes \vec{u}_{2}^{*}-\frac{\partial W}{\partial w} \frac{\vec{w} \otimes \vec{w}^{*}}{w}+\right. \\
\left.+\left(\rho_{1} \frac{\partial W}{\partial \rho_{1}}+\rho_{2} \frac{\partial W}{\partial \rho_{2}}-W\right) I\right)=0 \\
\frac{\partial}{\partial t}\left(\frac{1}{2} \rho_{1}\left|\vec{u}_{1}\right|^{2}+\frac{1}{2} \rho_{2}\left|\vec{u}_{2}\right|^{2}+U\right)+\operatorname{div}\left(\rho_{1} \vec{u}_{1}\left(\frac{\left|\vec{u}_{1}\right|^{2}}{2}+\frac{\partial W}{\partial \rho_{1}}\right)+\rho_{2} \vec{u}_{2}\left(\frac{\left|\vec{u}_{2}\right|^{2}}{2}+\frac{\partial W}{\partial \rho_{2}}\right)-\right. \\
\left.-\frac{\partial W}{\partial w}\left(\vec{u}_{2} \otimes \vec{u}_{2}^{*}-\vec{u}_{1} \otimes \vec{u}_{1}^{*}\right)<\frac{\vec{w}}{w}>\right)=0
\end{gathered}
$$

where $\otimes$ denotes the tensor product and $I$ is the unit tensor.

The question of the hyperbolicity of the system $(1.2),(2.4)$ is of great interest. Unfortunately, the multi-dimensional case is not very simple and this is why we restrict our attention on the one-dimensional case. Moreover, to simplify the calculations, we consider the case (1.4) and linearize our system in the neighbourhood of the equilibrium state $\vec{u}_{1}^{0}=\vec{u}_{2}^{0}=0, \rho_{1}^{0}, \rho_{2}^{0}$. Straightforward calculations give the following result: if $\varepsilon\left(\rho_{1}, \rho_{2}\right)$ is a convex function and $a\left(\rho_{1}, \rho_{2}\right)>0$ then linearized at rest, the system $(1.2),(2.4)$ is hyperbolic.

\section{3 - The Rankine-Hugoniot conditions}

The non-linearity and hyperbolicity of the system (1.2),(2.4) imply the necessity to obtain Rankine-Hugoniot conditions across shocks. In multi-dimensional 
case the conservation laws $(1.2),(2.5),(2.6)$ are not sufficient to obtain the whole set of such relations. In what follows, we show that the additional jump conditions can be derived from the variational principle.

Let us define variations of particules deduced from the relation $\vec{x}=$ $\vec{\Phi}_{i}\left(\vec{X}_{i}, t, \varepsilon_{i}\right)$ and its inverse $\vec{X}_{i}=\vec{\Psi}_{i}\left(\vec{x}, t, \varepsilon_{i}\right)$. Here $\varepsilon_{i}, i=1,2$ are small parameters defined in a neighbourhood of zero. One defines virtual displacements $\delta_{i} \vec{x}$ and $\delta_{i} \vec{X}_{i}$ by

$$
\delta_{i} \vec{x}=\frac{\partial \vec{\Phi}_{i}}{\partial \varepsilon_{i}}\left(\vec{X}_{i}, t, 0\right), \delta_{i} \vec{X}_{i}=\frac{\partial \vec{\Psi}_{i}}{\partial \varepsilon_{i}}(\vec{x}, t, 0) .
$$

It is clear that $\delta_{i} \vec{X}_{i}=-F_{i}^{-1}<\delta_{i} \vec{x}>$, where $F_{i}=\frac{\partial \vec{x}}{\partial \vec{X}_{i}}$. For any variable $\alpha_{i}(\vec{x}, t)$ we define their eulerian perturbations $\hat{\alpha}_{i}\left(\vec{x}, t, \varepsilon_{i}\right)$ and lagrangian perturbations $\tilde{\alpha}_{i}\left(\vec{X}_{i}, t, \varepsilon_{i}\right)=\alpha_{i}\left(\vec{\Phi}_{i}\left(\vec{X}_{i}, t, \varepsilon_{i}\right), t, \varepsilon_{i}\right)$. The variations $\hat{\delta}_{i} \alpha_{i}$ and $\tilde{\delta}_{i} \alpha_{i}$ of $\alpha_{i}$ are defined by

$$
\hat{\delta}_{i} \alpha_{i}=\frac{\partial \hat{\alpha}_{i}}{\partial \varepsilon_{i}}(\vec{x}, t, 0), \quad \tilde{\delta}_{i} \alpha_{i}=\frac{\partial \tilde{\alpha}_{i}}{\partial \varepsilon_{i}}\left(\vec{X}_{i}, t, 0\right) .
$$

It follows from the above definitions that

$$
\hat{\delta}_{i} \alpha_{i}=\tilde{\delta}_{i} \alpha_{i}-\frac{\partial \hat{\alpha}_{i}}{\partial \vec{x}}<\delta_{i} \vec{x}>
$$

In particular,

$$
\hat{\delta}_{i} \rho_{i}=-\operatorname{div}\left(\rho_{i} \delta_{i} \vec{x}\right), \hat{\delta}_{i} \vec{u}_{i}=\frac{d_{i}}{d t} \delta_{i} \vec{x}-\frac{\partial \vec{u}_{i}}{\partial \vec{x}}<\delta_{i} \vec{x}>
$$

We define

$$
\delta_{i} I=\lim _{\varepsilon_{i} \rightarrow 0} \frac{I\left(\varepsilon_{i}\right)-I(0)}{\varepsilon_{i}} .
$$

Then

$$
\delta_{i} I=\int_{t_{1}}^{t_{2}} \int_{\mathcal{D}}\left(\hat{\delta}_{i} \rho_{i}\left(\frac{\left|\vec{u}_{i}\right|^{2}}{2}-\frac{\partial W}{\partial \rho_{i}}\right)+\rho_{i} \vec{K}_{i}^{*} \hat{\delta}_{i} \vec{u}_{i}\right) d \vec{x} d t .
$$

It follows from (3.3) (3.4) that

$$
\delta_{i} I=\int_{t_{1}}^{t_{2}} \int_{\mathcal{D}}\left(-\operatorname{div}\left(\rho_{i} \delta_{i} \vec{x}\right)\left(\frac{\left|\vec{u}_{i}\right|^{2}}{2}-\frac{\partial W}{\partial \rho_{i}}\right)+\rho_{i} \vec{K}_{i}^{*}\left(\frac{d_{i}}{d t} \delta_{i} \vec{x}-\frac{\partial \vec{u}_{i}}{\partial \vec{x}}<\delta_{i} \vec{x}>\right)\right) d \vec{x} d t
$$




$$
\begin{gathered}
=\int_{t_{1}}^{t_{2}} \int_{\mathcal{D}}\left(-\rho_{i} \delta_{i} \vec{x}^{*}\left(\vec{K}_{i t}+\frac{\partial \vec{K}_{i}}{\partial \vec{x}}<\vec{u}_{i}>+\frac{\partial \vec{u}_{i}^{*}}{\partial \vec{x}}<\vec{K}_{i}>+\nabla^{*}\left(\frac{\partial W}{\partial \rho_{i}}-\frac{\left|\vec{u}_{i}\right|^{2}}{2}\right)\right)+\right. \\
\left.+\frac{\partial}{\partial t}\left(\rho_{i} \delta_{i} \vec{x}^{*} \vec{K}_{i}\right)+\operatorname{div}\left(\rho_{i} \vec{u}_{i}\left(\delta_{i} \vec{x}^{*} \vec{K}_{i}\right)+\rho_{i} \delta_{i} \vec{x}\left(\frac{\partial W}{\partial \rho_{i}}-\frac{\left|\vec{u}_{i}\right|^{2}}{2}\right)\right)\right) d \vec{x} d t .
\end{gathered}
$$

As a consequence, we obtain equations of motion (2.4) and the jump conditions:

$$
\left[\rho_{i}\left(\vec{n}^{*} \delta_{i} \vec{x}\right)\left(\frac{\partial W}{\partial \rho_{i}}-\frac{\left|\vec{u}_{i}\right|^{2}}{2}\right)+\rho_{i}\left(\vec{n}^{*} \vec{u}_{i}\right)\left(\vec{K}_{i}^{*} \delta_{i} \vec{x}\right)-D_{n} \rho_{i}\left(\vec{K}_{i}^{*} \delta_{i} \vec{x}\right)\right]=0
$$

where $\vec{n}$ is the unit normal vector to the shock surface and $D_{n}$ is the normal velocity of the shock. Since $\delta_{i} \vec{x}$ is not continuous across the shock, it is not straightforward to obtain Rankine- Hugoniot conditions in terms of desired quantities. Nevertheless, taking into account that across the shock

$$
\left[\rho_{i} F_{i}^{*}<\vec{n}>\right]=0,
$$

such difficulties can be overcame. We finally obtain from (3.5):

$$
\begin{gathered}
{\left[\frac{\partial W}{\partial \rho_{i}}-\frac{\left|\vec{u}_{i}\right|^{2}}{2}+\vec{K}_{i}^{*} \vec{u}_{i}-D_{n}\left(\vec{K}_{i}^{*} \vec{n}\right)\right]=0,} \\
{\left[\vec{K}_{i}-\left(\vec{K}_{i}^{*} \vec{n}\right) \vec{n}\right]=0 .}
\end{gathered}
$$

In the limiting case when the velocities of the components coincide, these conditions reduce to the conservation of the tangential component of the velocity and the conservation of the Bernoulli constant across the shock. We note also that conditions (3.6), (3.7) are obtained from the variational principle (1.1) without any assumption on the flow properties. But it can be shown directly that they correspond to the jump conditions for the additional conservation laws admitted by the system $(1.2),(2.4)$ :

$$
\operatorname{rot} \vec{K}_{i}=0, \quad \frac{\partial \vec{K}_{i}}{\partial t}+\nabla^{*}\left(\frac{\partial W}{\partial \rho_{i}}-\frac{1}{2}\left|u_{i}\right|^{2}+\vec{K}_{i}^{*} \vec{u}_{i}\right)=0 .
$$

The conservation laws (2.5), (2.6) also imply momentum balance and energy balance at the shock. Finally note that all possible jump conditions are of great interest but the correct choice of the jump conditions depends on the physics of the problem. 


\section{References}

Berdichevsky V.L. (1983) Variational principles of continuum mechanics. Moscow : Nauka, 1983.

Bedford A. \& Drumheller D.S. (1978) A variational theory of immiscible mixtures. Arch. Rat. Mech. Annal. 1978. Vol. 68. P.37-51.

Geurst J. A. (1985) Virtual mass in two-phase bubbly flow. Physica. A. 1985. Vol.129A. P.233-261.

Geurst J. A. (1986) Variational principles and two-fluid hydrodynamics of bubbly liquid / gas mixtures. Physica. A. 1986. Vol.135A. P.455-486.

Gouin H. (1990) Variational theory of mixtures in continuum mechanics. Eur. J. Mech, B / Fluids. 1990. Vol.9, No.5. P.469-491. 